\title{
DAYLIGHT VARIABILITY AND HUMAN PERCEPTION with reference to selected Contemporary Residential Spaces in Colombo, Sri Lanka
}

\author{
PATHIRAGE, L. Y. ${ }^{1} \&$ PERERA, N.G.R. ${ }^{2}$ \\ 1,2 University of Moratuwa, Moratuwa, Sri Lanka \\ 1lakminiyashoda@gmail.com, 2nareinperera@uom.lk
}

\begin{abstract}
Visual perception of a space is influenced by the composition and behaviour of light. Daylight as a natural element, creates different atmospheres in the same space by its variation of intensity, colour, and direction. It creates dynamic shadow patterns, fluctuating brightness and contrast levels. Although the capacity to create variation therefore contrast, is an important characteristic of daylight - it is often neglected in research, where comfort and visual performance take prominence. This is a research initiative to emphasise the value of daylight variation on spatial quality and human perception. The research adopts a two-step process, where, digital images are first mapped to generate contrast diagrams, and secondly, the generated digital images form the basis for a survey utilising a sevenpoint semantic differential scale, to explore the relationship between daylight variability and human perception. The temporal variation of daylight is explored by diagramming the spatial images at selected times of the day and year. The scope of the study is limited to selected contemporary residential spaces and focus on the manner of integrating daylight into spaces. Results show that a direct relationship between daylight variability and human perception for side lit spaces is not evident, yet it is evident in top lit options.
\end{abstract}

Keywords: Daylight variability, Human perception, Contrast, Daylight integration

\section{Introduction}

The most eloquent creations with light in the history of architecture have not been overly concerned with visual acuity and formal clarity. Throughout the ages and with a quiet, almost unspoken skill, masterful builders have sought to catch certain parts of the sky, and then carefully guide those oncoming rays into the spatial components and material fabric of buildings, so that some parts might darken and vanish, and others grow bright and shimmer. Light was projected, reflected, dimmed, focussed, diffused, and scattered not to expose the surface of things, but to bring into the deadened weight of the architecture a more enchanting and vibrant reality. (Perera, 1998).

The perception of space is directly connected to the way light integrates with it. What we see, what we experience and how we interpret the elements is affected by how light interacts with us and with the environment. Regarding architecture, in whatever dimension it can be analysed, either as space, as material or as colour, it is essentially dependant on the lighting situation that involves both the object and the observer. (Fontenelle, 2008).

Daylight as a natural source of lighting is highly variable, and it has become the basis for studies on threshold properties of lighting and its impact. The most obvious aspect of daylight is its capacity for change, leading to a great variety of appearance \& quality of the daylit interior (Philips, 2004). Although there is an understanding of the minimum amount of illumination that is required for the human eye to perform visual tasks, there is little consensus on how much contrast or brightness makes that space more or less exciting. (Rockcastle, 2011)

The capacity to create variation - therefore contrast, an important characteristic of daylight - is 
often neglected in research, where comfort and visual performance take prominence.

In this context, the primary aim of this research is to explore and emphasise the value of daylight variation - analysed in terms of contrast - on spatial quality and human perception.

The research is designed in two steps. The first step maps and analyses spaces in terms of contrast. The objective of the step is to establish a measured contrast intensity that informs and compares the next step in the process - the perception survey. In the next step, a perception survey is conducted, involving thirty participants, that rate the digital images presented to them in terms of the level of contrast, uniformity, variation, simplicity, excitement, and stimulation. The objective of this step is to explore the correlation between contrast intensity and visual perception of the observer.

The scope of the study is limited to contemporary residential spaces in Colombo, Sri Lanka. The residential typology is chosen due its increased exploration of light as a key compositional element. Two case studies are chosen, and the selected spaces explore side lighting and top lighting strategies, as a further limitation.

Results and analyses encompass a comparative data collation for fifty-six digital images. The images represent seven times of a particular day, and eight selected days of the year, to explore the temporal nature of the daylit space.

\section{Literature Review}

Light is a fundamental element of architecture; it interacts with the space, affecting the way we perceive it. The way lighting acts can change the spatiality, the atmosphere, and the visibility. The lit environment deals with brightness, shadows, lighting distribution, colour and many other aspects that influence our visual experience and plays with our mood. (Fontenelle, 2008).

\subsection{HUMAN PERCEPTION ON DAYLIGHT AND ITS VARIATIONS}

Lighting is not only an essential requirement, but also the medium by which the human being is able to see. The way light is distributed in spaces and its intensity influences visual perception. Good lighting design requires the enhancement of the visual experience, which promotes an effective working ability as well as a feeling of well-being in a particular environment, while at the same time enhancing the aesthetic qualities of the space.

The light changes continuously over time. Such fluidity gives life to the space as if it were a living object. Thus, the light gives different feelings to the same physical space as time changes, and such altered feelings affect the sentiment of people. The space itself is in three-dimensions. However, the light enables people to experience it in four-dimensions by embracing the dimension of time when it is joined with the light. $(\mathrm{Kim}, 2000)$

\subsection{BRIGHTNESS AND CONTRAST}

Brightness and contrast are two basic components in visual perception which are often mentioned together. Brightness is the perceived illuminance as the result of direct and reflected light. Human eyes always capture the relative brightness which is known as contrast. In simple words, contrast is the balance between light and darkness. The eye's ability to perceive those differences and the brain's ability to extract that information are essential in order to understand the depth and complexity of the surrounding. (Rockcastle, 2011). (Figure 1).

\subsection{CONTRAST METRICS}
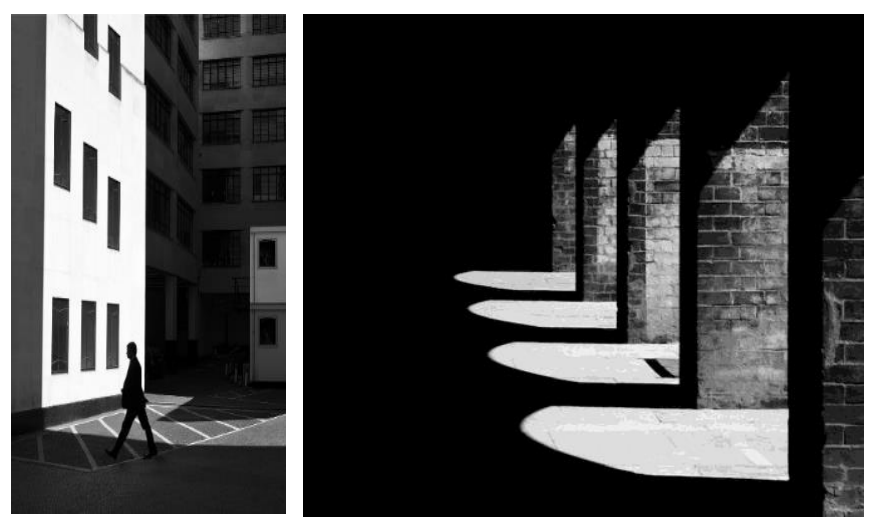

Figure 1, Relationship of light and dark in the built environment

(Source: creativeboom)

Perceptual contrast of an image depends on the observer's vision and experience. Perceived contrast is influenced by the surrounding and the spatial arrangement of the image. Parameters such as resolution, 
viewing distance, lighting conditions, image content, memory, and colour, affect how the observer perceives contrast. (Simone et al., 2012) The same authors divide the existing contrast measures into two categories, as global contrast measures and as local contrast measures.

Global measures are based on the claim that the human visual system depends on the absolute illuminance and not on its local variations (Simone et al., 2012). As an example, the Michelson formula ( $\mathrm{C}^{\mathrm{M}}$ ) measures the contrast as the deviation ranging of maximum luminance ( $L \max$ ) and minimum luminance (Lmin).

$$
\mathrm{C}^{\mathrm{M}}=\frac{\left(\mathrm{L}_{\max }\right)-\left(\mathrm{L}_{\min }\right)}{\left(\mathrm{L}_{\max }\right)+\left(\mathrm{L}_{\min }\right)}
$$

As per this method, different images with same maximum and minimum luminance can produce the same contrast measurement, which is criticised by the Simone, who states that "two single points of extreme brightness or darkness cannot determine the measure of contrast in the whole image".

Local contrast measures are developed to overcome the limits of global measures. They all compare illuminance in pixels or desired points. Rockcastle \& Anderson, 2014, compares the two methods of contrast measures using the same number of black and white pixels (15 from each) with three different arrangements (Figure 2).

Composition A

30 pixels: 15 black (0) and 15 white (255)

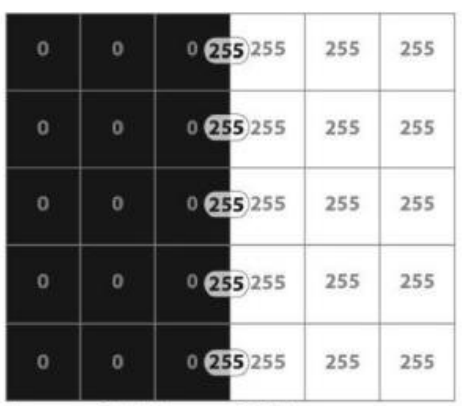

Average Brightness $=127.5$

Standard Deviation $=129.7$

Spatial Contrast $\approx 10 \%$
Composition B

30 pixels: 15 black (0) and 15 white (255)

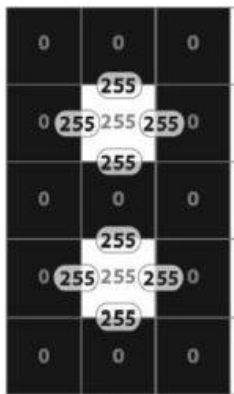

Average Brightness $=127$

Standard Deviation $=129.7$

Spatial Contrast $\approx 50 \%$

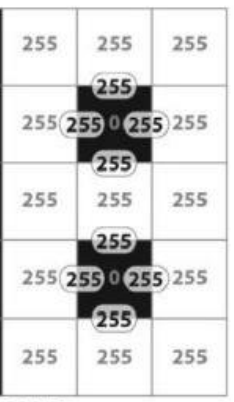

Composition C - MAXIMUM

30 pixels: 15 black $(0)$ and 15 white (255)

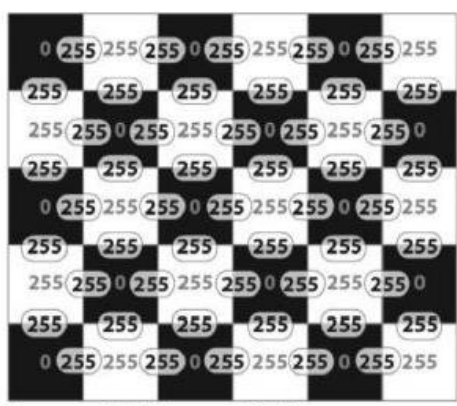

Average Brightness = 127.5

Standard Deviation $=129.7$

Spatial Contrast $=100 \%$

Figure 2, Image contrast of three compositions with same number of black \& white pixels

(Source: Rockcastle \& Anderson.)

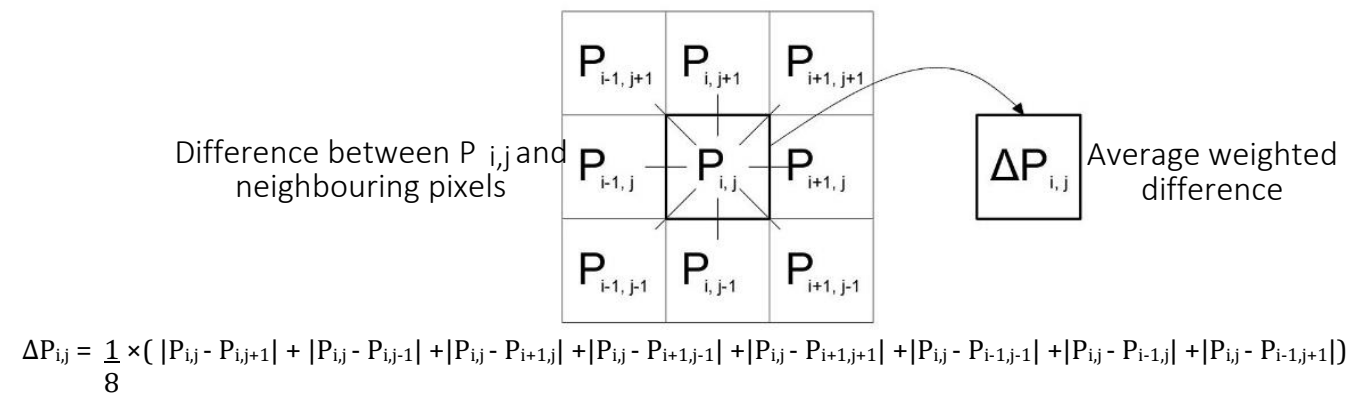

Figure 3, Calculation of RAMMG method

(Source: Rockcastle \& Anderson.)

The RAMMG method has been used as a local contrast measure, which gives a different value for three examples, while the Michelson formula produces the same value (Figure 3). RAMMG, a contrast algorithm developed by (Rizzi, et al., 2004), applies a multi-level approach to compute mean local pixel variations across a subsampled pyramid structure, to account for perceived differences in brightness across multiple image resolutions. (Rockcastle \& Anderson, 2015). 


\section{Method}

The research method encompasses two main components.

1. Daylight variation and contrast

2. Contrast variation and human perception

\subsection{STEP 01: DAYLIGHT VARIATION AND CONTRAST}

\subsubsection{Categorisation of naturally lit spaces and case study selection}

The performance of daylight depends on the way that daylight is enters to the space. The primary way of introducing natural light into interior is through a window. The design \& shape of the window has evolved over the centuries, but the primary purpose remains the same. Two types of fenestrations are used in building design and defined as, side lights \& roof lights. (Philips, 2004).

Since Sri Lanka is located closer to the equator, natural light is available throughout year. Two contemporary Architect designed residences in the Colombo region were selected as case studies.

The spaces were categorised according to the type of fenestration.
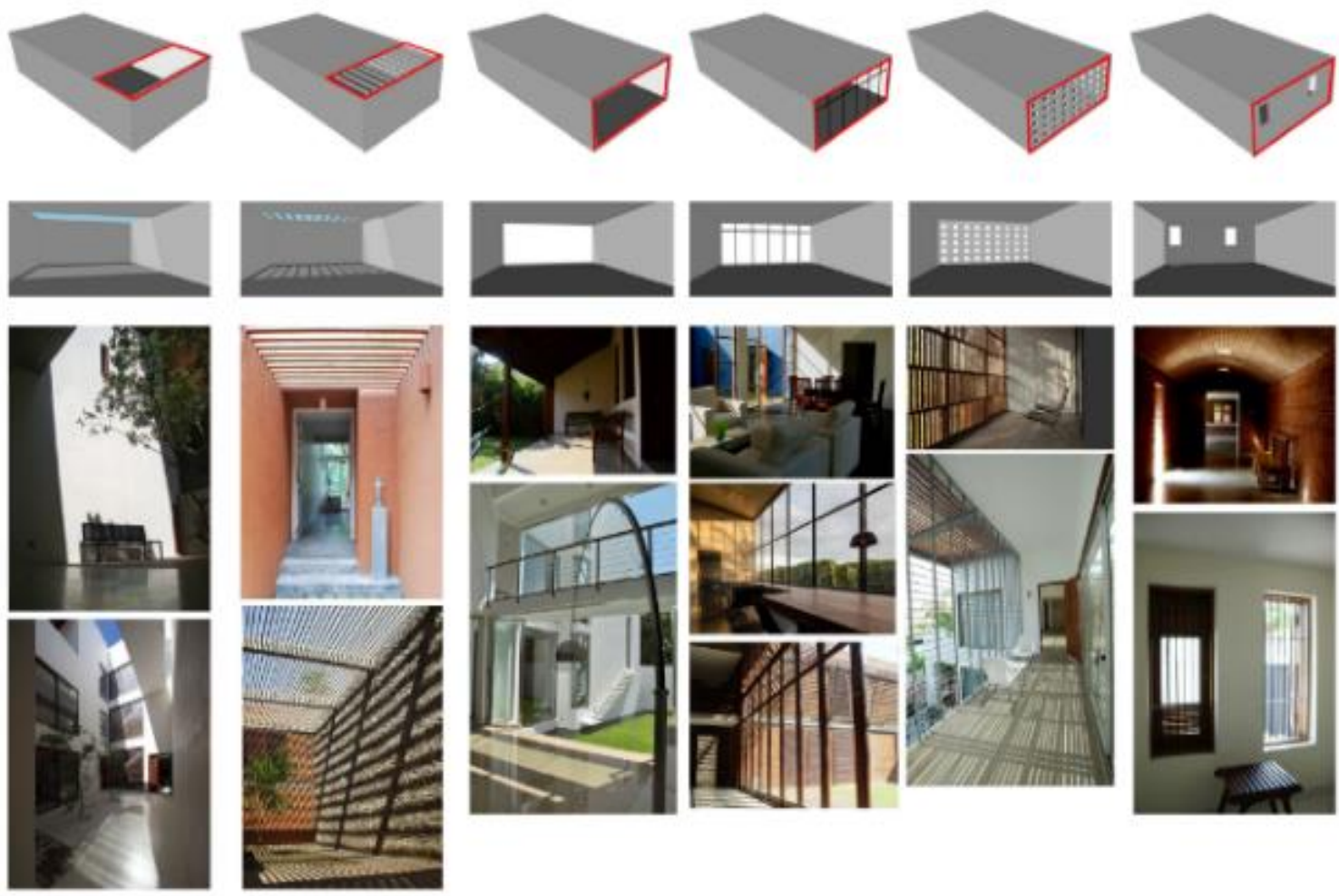

Figure 4, Categorising of spaces according to the type of opening (Source: Author)

1. Completely open top lit spaces

2. Top lit spaces with repetitive obstructions

3. Completely open side lit spaces

4. Side lit spaces with minimum obstructions

5. Side lit spaces with repetitive openings

6. Side lit spaces with thick openings

Although, side and top opening options are extensive in its permutations and combinations, for the purpose of in-depth analysis of spaces, two residences designed by Archt. Perera and Archt. Horombuwa were explored and daylit spaces were selected. The selection, though random in terms of contemporary residences, was based on data availability and the Architect's conscious utilisation of daylight for architectural effect. Both case study residences were recognised by the Sri Lanka Institute of Architects for 
Design Excellence. The selected spaces within the two case studies falls into the identified Category 2 - Top lit spaces with repetitive obstructions, and Category 4 - Side lit spaces with minimum obstructions.

\subsubsection{Computer simulation protocol and Contrast Intensity Mapping}

Daylighting analysis methods have evolved over time and two primary types are defined, as physical modelling and computer-based simulations. Since daylighting performance cannot be predicted like in artificial lighting, simulated analysis systems are used to evaluate the performance of daylight before the completion of the building. Illuminance levels and spatial variations can be studied and developed prior to the finalisation of the design. Yet, these methods are not completely dependable. As Kensek \& Suk state, "It is not easy to get accurate, predictable or often matching values between techniques due to the multitude of variables, including sky selection." (Kensek \& Suk, 2011).

In this context the primary mode of mapping of spaces and communication adopt computer simulations. Utilising the modelling software, Autodesk 3ds Max, (formerly 3D Studio and 3D Studio Max), a professional 3D computer graphics program. Each image of a space, on the 56-point temporal matrix is rendered and mapped for contrast intensity utilising the NVIDIA Mental Ray for Autodesk 3ds Max plugin. The Autodesk 3ds Max lighting analysis tool produces an intensity map for the camera view to the given pixel gap. (Figure 5). Using the RAMMG method (detailed in section 2.3), contrast of each point is calculated related to the eight points around it.
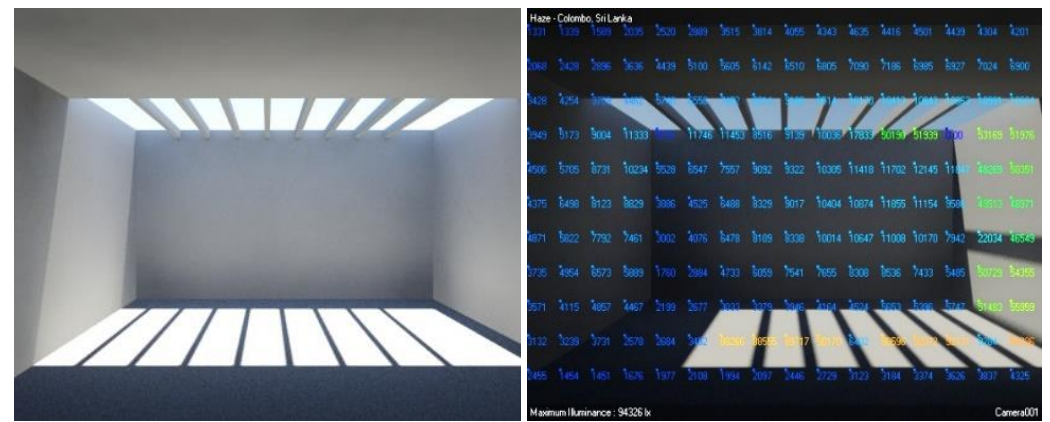

Figure 5, Rendered Image, and Intensity Map produced by Autodesk 3ds Max (Source: Author)

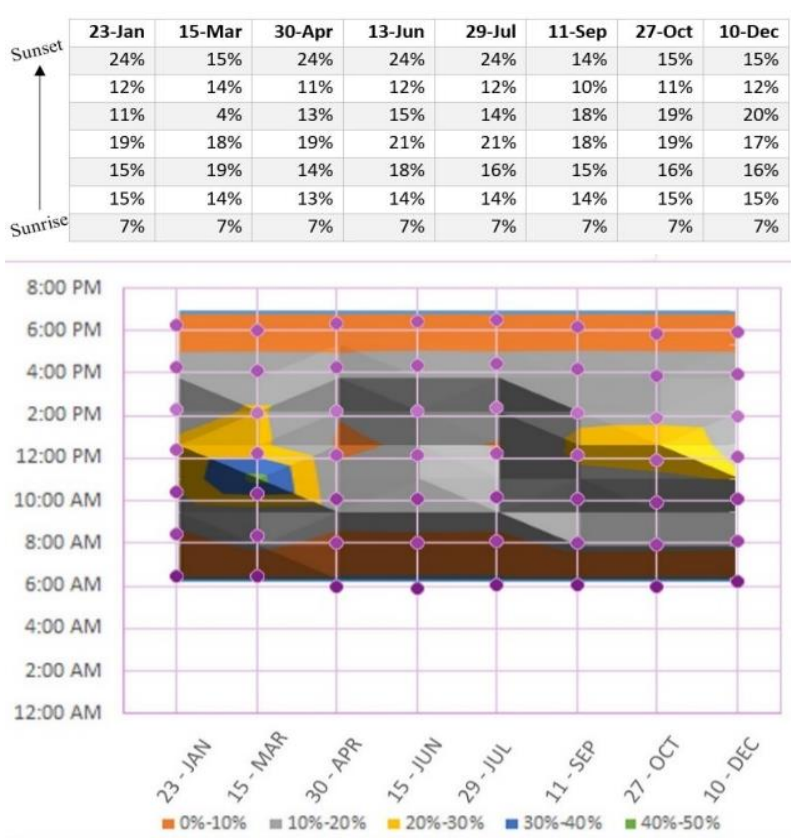

Figure 6, Mean value of contrast and Contrast diagram

(Source: Author)

Eight days of the year with equal number of days between them were selected. Seven times of each day were selected considering the time of sunrise and sunset. These days and time-of-day establish the temporal matrix utilised for the next stage of the research process. The rendered images presented as a 
matrix is shown in Figure 5. These images form the format used to communicate the spatial qualities and variances to the participants.

Thereafter, a mean value of contrast intensity of each image is calculated and communicated as assigned colours, depicting intensity as a percentage, as shown in Figure 6.

\subsection{STEP 02: CONTRAST VARIATION AND HUMAN PERCEPTION}

\subsubsection{Survey Design}

To explore the contrast variation and human perception component of the research, the approach utilised by Rockcastle \& Anderson, 2015, was adopted, where "for each space, the subjects were shown an image and asked to rate the daylight composition using the following seven-point semantic differential scales: low contrast to high contrast, uniform to non-uniform, unvaried to varied, simple to complex, calming to exciting, sedating to stimulating". (Rockcastle \& Anderson, 2015) (Figure 7). Numerous studies have employed the use of these scales to conduct daylight quality research in real spaces, and for simulated or photographed views (Newsham, et al., 2002; Newsham, et al., 2005; Vogels, 2008; Demers, 2007) (as cited in Rockcastle \& Anderson, 2015).

The perception of thirty (30) subjects were surveyed, to explore the correlation between contrast intensity and visual perception of the observer. The selected participants were between the ages of 21 to 24 , and female, who have no relation to the field of lighting design or analysis. The limitation of the participants was chosen such that the sampling can be focussed as possible and also due to the nature of the perception study, where personal factors can affect perception.

\subsubsection{Analysis Protocol}

$\begin{array}{rllllllll}\text { How would you rate the daylight compostion in this image? } & \\ \text { low contrast } & \bigcirc & 0 & 0 & 0 & 0 & 0 & 0 & \text { high contrast } \\ \text { uniform } & 0 & 0 & 0 & 0 & 0 & 0 & 0 & \text { non-uniform } \\ \text { unvaried } & 0 & 0 & 0 & 0 & 0 & 0 & 0 & \text { varied } \\ \text { simple } & \bigcirc & 0 & 0 & 0 & 0 & 0 & 0 & \text { complex } \\ \text { calming } & \bigcirc & 0 & 0 & 0 & 0 & 0 & 0 & \text { exciting } \\ \text { sedating } & 0 & 0 & 0 & 0 & 0 & 0 & 0 & \text { stimulating }\end{array}$

Figure 7, The rating scale used for the survey.

(Source: Rockcastle \& Anderson, 2015)

The analytical study attempts to explore the relationship between spatial quality and human perception by using contrast as a research tool. A comparison is expressed between the measured contrast (quantitative data) and recorded human perception (qualitative data) to achieve the task.

Daylight variability and contrast level of case study spaces are explored in two levels.

Firstly, the Rendered images of 56 instances were arranged sequentially to observe how the same space changes its spatial quality during a year. The use of digital tools is a distinct advantage in this instance, where the sequence of images was generated within a short period of time.

Thereafter, in the second step, six images were selected from each case study (from within the 56 images that were generated in the previous step). The selection of images is taken as a limitation of scope, where a survey utilising all 56 images was deemed to be excessive. Further, the levels of contrast were too similar to generate a perception response. The selected images represent different and distinct contrast levels, and represents a particular time of day and day of a year. These images established the basis of the survey, ensuring variation of contrast and temporality. The survey was conducted using three sets of images consisting of four images - two from each case study.

The survey results are then correlated to the selected image and its mean contrast intensity. 


\section{Results and Discussion}

\subsection{CASE STUDY 1 - "ENIGMA" BY ARCHT. NAREIN PERERA}

The space used for evaluation is categorised as a "Side lit space with minimum obstructions". The primary opening faces to the South-East direction. Clear shadow patterns are visible only for few months of the year and spatial contrast is high during that period. (See Figure 8)

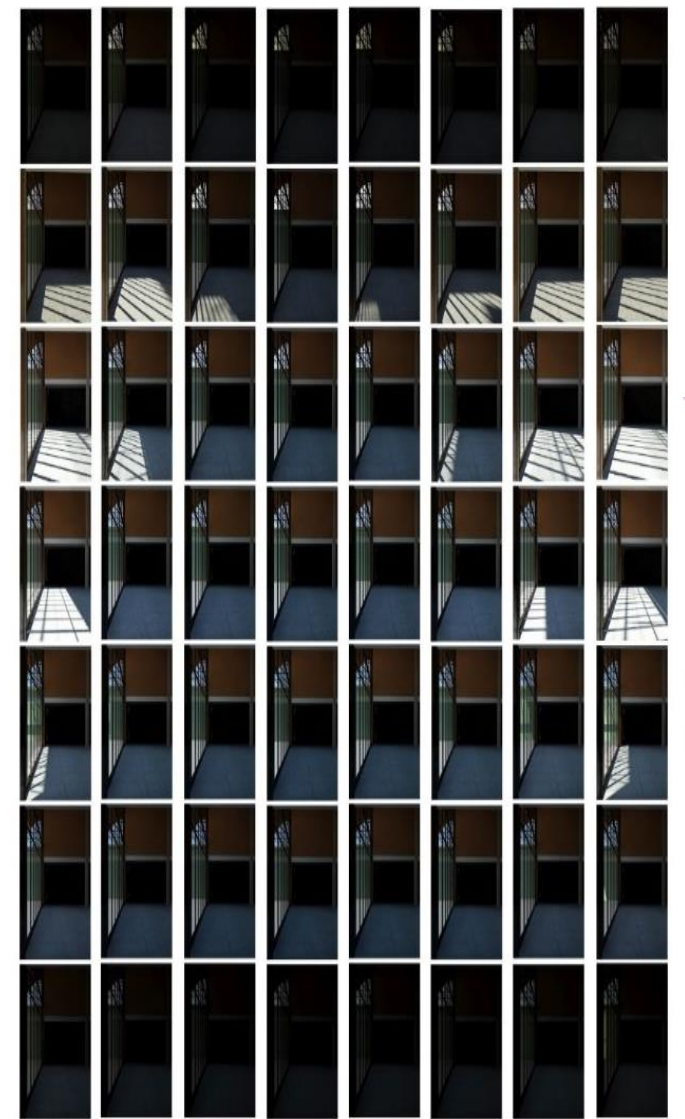

$10 \%$
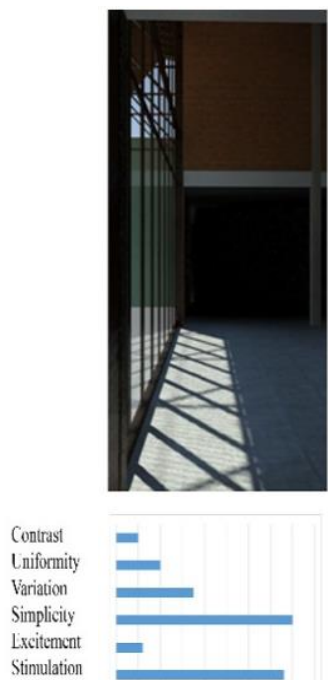

$6.6 \%$

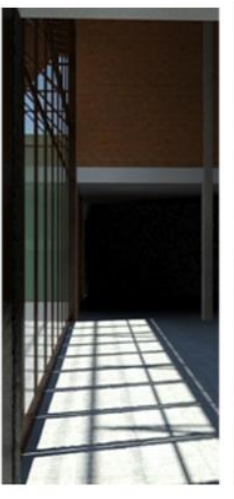

Figure 8. CASE STUDY 1 - Annual temporal renderings (top left), calculated contrast (top right),

\begin{tabular}{|c|c|c|c|c|c|c|c|}
\hline 23-Jan & 15-Mar & 30-Apr & 13-J & 29-Jul & 11-Sep & $27-0$ & 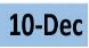 \\
\hline $1.1 \%$ & $1.1 \%$ & $9.0 \%$ & $0.9 \%$ & 0.9 & & $1.0 \%$ & $1.0 \%$ \\
\hline 8.4 & & & & 8 & $10.6 \%$ & $12.2 \%$ & $12.2 \%$ \\
\hline $10.6 \%$ & $10.0 \%$ & $5.9 \%$ & & & & 15.1 & $14.5 \%$ \\
\hline $11.2 \%$ & $5.8 \%$ & $5.8 \%$ & 5. & 5.7 & $5.8 \%$ & 14.0 & $16.8 \%$ \\
\hline $9.2 \%$ & $4.7 \%$ & $4.7 \%$ & 4.79 & 4.6 & 4.5 & $4.5^{\circ}$ & $10.8 \%$ \\
\hline 5.2 & & 5.3 & & & & 5.4 & $5.2 \%$ \\
\hline $2.2 \%$ & $.2 \%$ & $2.3 \%$ & $2.3 \%$ & $2.3 \%$ & $2.2 \%$ & $2.2 \%$ & $2.2 \%$ \\
\hline
\end{tabular}

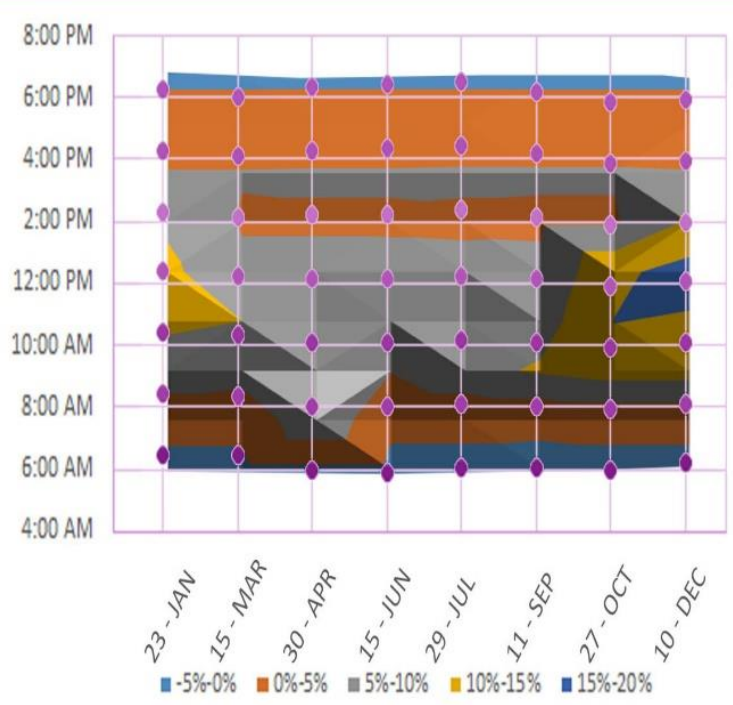

$5.7 \%$

$9.2 \%$

$12.2 \%$
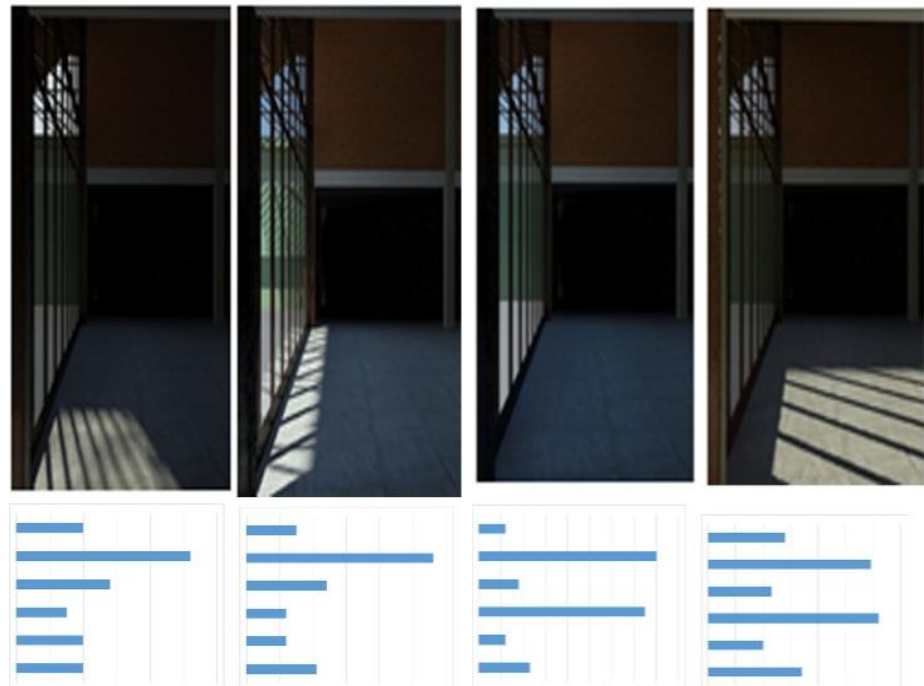
contrast diagram (middle right) and Recorded perception (bottom)

(Source: Author)

For the side lit space, the perception survey shows that the measured value of contrast does not correspond to the perceived 'contrast' level for the observer. However, it is seen that the corelation of the 
pattern of shadow takes prominence. This is also seen in correlation to the 'uniformity', where the observers see the space as 'non-uniform' corresponds to the times of the year where shadows, and therefore contrast is low. This seems to be contradictory, as also seen in the relationship between 'uniformity' and 'variation', where the observers perceive that the highly non-uniform spaces offer low variety. The perception of the level of 'stimulation' the space provides correlates well with measured contrast values. (Figure 8). The measured high contrast patterns correspond to a 'calming' effect (as opposed to 'exciting') in the survey results.

Overall, the semantic properties of a side lit space do not strongly correlate to measured daylight variability. They seem to be governed by other factors, like the presence of shadows on surfaces and the patterns they paint.

\subsection{CASE STUDY 2 - “MINIMALIST HOUSE” BY ARCHT. CHANNA HOROMBUWA}

The space selected for evaluation falls within Category 2 - Top lit spaces with repetitive obstructions. The considered space receives a good amount of daylight throughout the year. The material and colour selection enhances the reflection of light within the space. Sharp shadows are created during the daytime hours. The contrast diagram shows a high contrast during the middle months of the year.

For the top lit space, the perception survey shows a strong correlation between measured and perceived 'contrast'. (See Figure 9.) Similarly, the 'non-variability' within the perceived space, and the ability to create 'excitement' within it, is positively correlated to contrast. Therefore, high contrast spaces are perceived as 'non-variable' and 'exciting'. The aspect of 'uniformity' does not show a pattern, even in consideration of the presence of shadows on the surfaces. Similarly, in consideration if the space
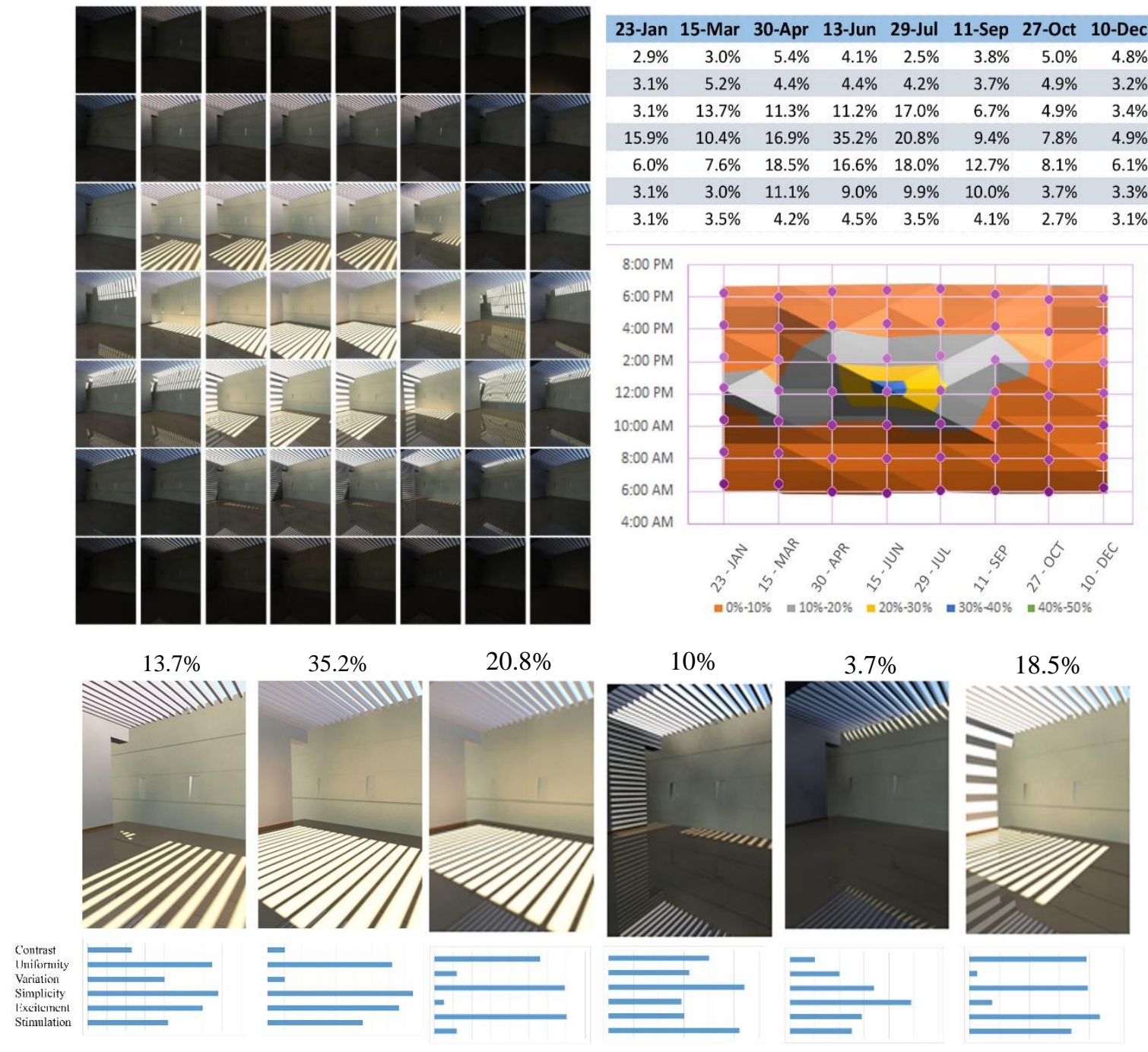

$18.5 \%$

Figure 9. CASE STUDY 2 - Annual temporal renderings (top left), calculated contrast (top right), contrast diagram (middle right) and Recorded perception (bottom)

(Source: Author) 
demonstrates 'simplicity' or 'complexity', and if the space is 'sedating' or 'stimulating', does not show a pattern in the perception survey. (Figure 9). Therefore, it is ascertained that the contrast intensity of the top lit space evaluated is perceived to be correlated to only particular parameters explored in the semantic survey.

\subsection{OVERALL CORRELATION OF PERCEPTION PARAMETERS}

The collected data is analysed for both the side lit and the top lit case studies. Overall correlation of recorded perception is compared for each parameter explored in the perception survey against the contrast intensity value communicated by the images. The objective is to ascertain if a relationship is evident between the measured contrast intensity and the parameters, independent of the manner in which the spaces are lit.

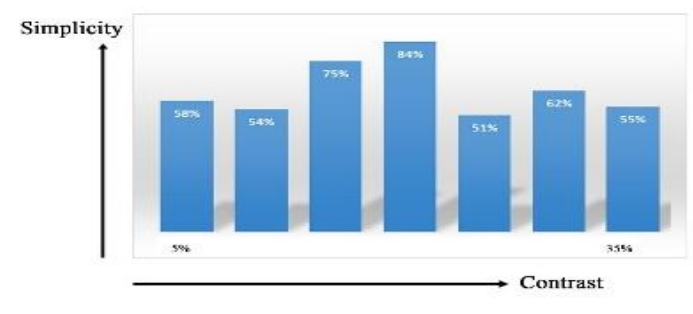

\subsubsection{Measured contrast vs Simple - Complex}

The graph shows that the perception of 'simplicity' or 'complexity' within the space varies with varied contrast intensity, and therefore does not show a direct relationship. Users perceive the space as more 'complex' at a certain threshold value.

\subsubsection{Measured contrast vs Calming - Exciting}

The results show a relatively clear pattern, where, at low contrast intensity values, the spaces are perceived as 'calming'. For the spaces to create excitement the temporal nature of daylight, the shadows it creates needs to be emphasised by contrast.

\subsubsection{Measured contrast vs Sedating - Stimulating}

Contrast driven properties do not correspond to a notion of whether a space is 'sedating' or 'stimulating'. Thus, it is deemed that other factors need to play a role in its spatial configuration.

\subsubsection{Measured contrast vs Uniform - Non-Uniform}

'Non-uniformity' of space is experienced with an increase in contrast. With contrast in lighting the ability of surfaces and materials to demonstrate their imbued properties are heightened. Therefore, the degree of detail is better perceived.

\subsubsection{Measured contrast vs Unvaried - Varied}

Although it can be expected that the level variation in the space will be heightened by the level contrast, the perception study shows that a definite pattern is not evident.
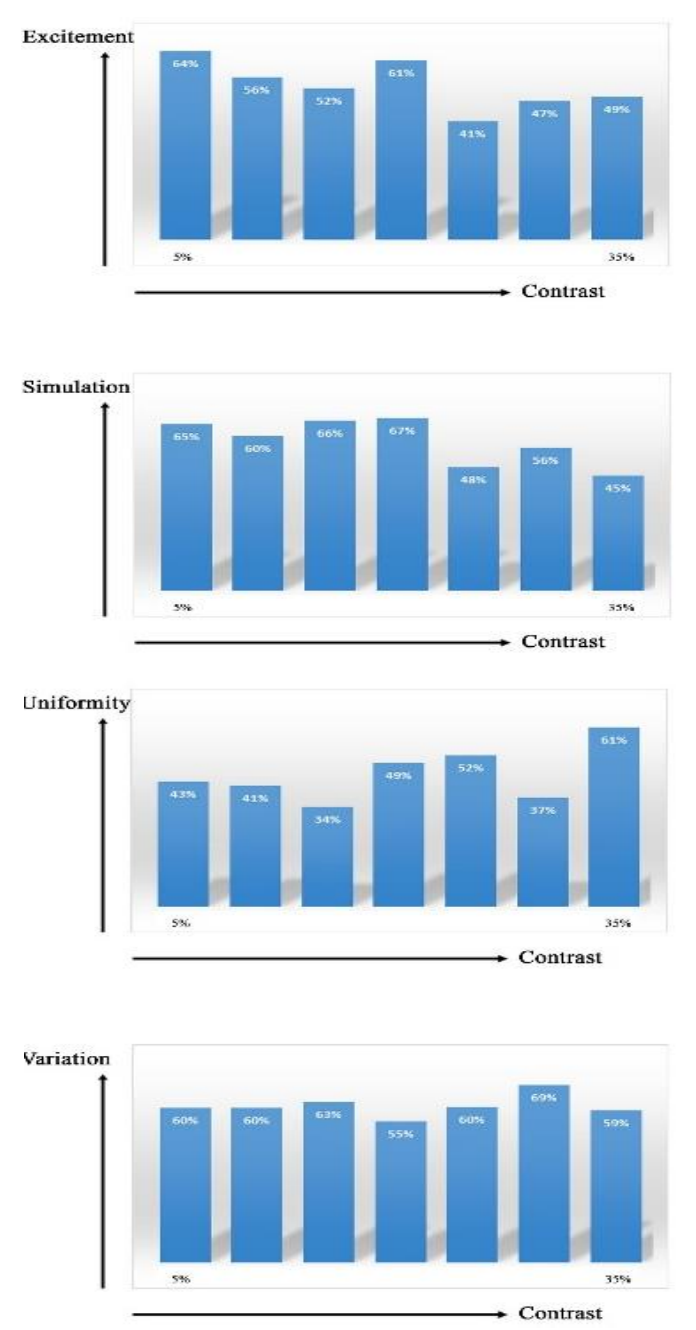

Figure 10, Overall correlation of perception parameters (Source: Author)

\section{Conclusion}

\subsection{KEY FINDINGS AND IMPLICATIONS FOR DESIGN}

5.1.1 For the side lit space, the measured value of contrast does not correlate to the perception of contrast for the observer.

Daylight variability perception for the side lit space did not show definite patterns. It is deemed that unlike the top lit space, shadow and defining boundaries of light and shadow elements were not as defined. 
Therefore, in image comparison by the observer, the differentiating factors were limited. Further, with the selected orientation, the ability of the side lighting aperture to admit light into a space is limited, especially direct sunlight components that create shadow.

For design and daylight integration for effect, the lesson here is that, in the exploration of a side lighting application, the designer needs to create an envelope of space, albeit with light. The light entering an interior space needs to illuminate, create patterns, and embrace the temporal character of light. Side openings need to be oriented, sized and placed such that either horizontal and vertical surfaces - or both of the physical envelope of a space is illuminated with pattern and / or intensity. Patterns created by elements included in the fenestration detailing will help create shadow and therefore interest.

\subsubsection{For the top lit space, the measured value of contrast strongly correlates to the perception of contrast for the observer.}

The top lit space effectively characterises the intensity and temporal qualities of the daylight integrated. This is affected by the repetitive elements within the opening (pergolas) and adjacent vertical surfaces that can 'catch' shadow. The top lit option can be more easily oriented, given the Sun's path over Sri Lanka is high in the sky, throughout the year. Even where the direct sun is shaded for solar heat gain prevention, the alternate mode of integrating daylight is effective because of the brightness of the sky for Sri Lanka.

Utilising top lighting strategies for light and therefore architectural effect has potential. With clear definition of strategy and appropriate design of the interior envelope to receive the positive elements of the integration, the space can be meaningful.

\subsection{LIMITATIONS AND DIRECTIONS FOR FUTURE STUDY}

The study utilised digital images and mapping techniques to measure and research the variability of daylight and its perception. Although a well-developed and utilised method was adopted, the greatest limitation is seen as the difference between experiencing a space physically and viewing it digitally, especially for communicating architectural effect. The challenges posed by the temporal nature and range of study, renders it impractical to be experienced physically. Future research needs to attempt to increase the understanding of the space the observer is expected to experience. Careful choice of image, that portrays architectural space rather than a mere graphic image can help.

Section 2 of this paper defined numerous ways of integrating light through openings. An expansion of the scope of study to include other typologies is encouraged.

Data collation and analysis was done graphically by means of graphs and bar charts. A more indepth statistical study with a larger data set and adoption of multiple regression and corelation between variables will help show patterns that are not evident at this stage.

\subsection{CONCLUSION}

Daylight; as a combination of direct sunlight and diffused skylight, ensure the vision and wellbeing of the user. The variation of daylight creates dynamic effect in a space without much effort. The research is an attempt to highlight and appreciate its variable quality. The key objective was to research on how human perception changes with the temporal variation of daylight.

Contrast is used as a tool to build up a relationship between daylight integration and human perception, primarily for architectural effect. Though it does not show a direct relationship for side lit spaces, it is strongly correlated to top lit options.

The correct and conscious integration of daylight into architectural space is essential to create spaces that convey meaning by utilising contrast to communicate uniformity, variability, simplicity or complexity, creating spaces that are calming or exciting, and that can sedate or stimulate the user. Thus, create an 'architecture in light' that ensures emotional and physical wellbeing of the occupant.

\section{References}

Fontenelle, C.V., 2008. The importance of lighting to the experience of architecture. Architecture Quality Issues, KTH Royal Institute of Technology, Stockholm, Sweden, pp.1-14. 
Kensek, K. and Suk, J.Y., 2011. Daylight Factor (overcast sky) versus Daylight Availability (clear sky) in Computerbased Daylighting Simulations. Journal of Creative Sustainable Architecture \& Built Environment, 1, pp.3-14.

Kim, K.J. (2000) A on the Variation in Meaning of Architectural Light Since Modern Architecture. Journal of Korean Institute of Interior Design, 25, 213-220.

Perera, N.G.R., 1998. "Light and its architectural effects”: with special reference to Buddhist Architecture of the Kandyan period. (Undergraduate Essay. University of Moratuwa)

Phillips, D., 2004. Daylighting: natural light in architecture. Routledge.

Rockcastle, S.F., 2011. Daylight variability and contrast-driven architectural effect (Doctoral Dissertation, Massachusetts Institute of Technology).

Rockcastle, S.F. and Andersen, M., 2015. Human perceptions of daylight composition in architecture: A preliminary study to compare quantitative contrast measures with subjective user assessments in HDR renderings. In Proceedings of the 14th International Conference of the International Building Performance Simulation Association (No. CONF).

Rockcastle, S. \& Andersen, M., 2014. Measuring the dynamics of contrast \& daylight variability in architecture: A proof-of-concept methodology. Building and Environment, Volume 81, pp. 320-333.

Simone, G., Pedersen, M. and Hardeberg, J.Y., 2012. Measuring perceptual contrast in digital images. Journal of Visual Communication and Image Representation, 23(3), pp.491-506.Aldeberky, A. A., 2004.The influence of highrise Buildings on the Environment. Centre of Planning andArchitectural Studies, Egypt. 